\title{
'In Vitro' Capacitation and Acrosome Reaction are Concomitant with Specific Changes in Mitochondrial Activity in Boar Sperm: Evidence for a Nucleated Mitochondrial Activation and for the Existence of a Capacitation-Sensitive Subpopulational Structure
}

\author{
L Ramió-Lluch ${ }^{1}$, JM Fernández-Novell ${ }^{2}$, A Peña ${ }^{1}$, C Colás ${ }^{3}$, JA Cebrián-Pérez ${ }^{3}$, T Muiño-Blanco ${ }^{3}$, A Ramírez $^{4}$, II Concha ${ }^{4}$, \\ T Rigau" ${ }^{1}$ and JE Rodríguez-Gil ${ }^{1}$ \\ ${ }^{1}$ Department of Animal Medicine and Surgery, School of Veterinary Medicine, Autonomous University of Barcelona, Bellaterra; ${ }^{2}$ Department of \\ Biochemistry and Molecular Biology, University of Barcelona, Barcelona; ${ }^{3}$ Department of Biochemistry and Molecular and Cell Biology, IUCA, \\ School of Veterinary Medicine, University of Zaragoza, Zaragoza, Spain; ${ }^{4}$ Biochemistry Institute and Animal Reproduction Institute, Austral \\ University of Chile, Valdivia, Chile
}

\section{Contents}

The main scope of this manuscript is to analyse the dynamics of mitochondrial activity in boar sperm subjected to 'in vitro' capacitation (IVC) and subsequent progesterone-induced 'in vitro' acrosome reaction (IVAR). This was determined after analysis of the rhythm of $\mathrm{O}_{2}$ consumption and concomitant changes in the mitochondria activity-specific JC-1 staining. Results showed that IVC, and especially IVAR, was concomitant with a peak in $\mathrm{O}_{2}$ consumption (from $1.61 \pm 0.08 \mathrm{nmol}$ $\mathrm{O}_{2} / \mathrm{min} / 10^{7}$ viable sperm at $0 \mathrm{~h}$ of incubation to $2.62 \pm$ $0.12 \mathrm{nmol} \mathrm{O}_{2} / \mathrm{min} / 10^{7}$ viable sperm after $5 \mathrm{~min}$ of IVAR induction). These results were accompanied by parallel changes in the mean intensity of JC-1 staining. Based on JC-1, mitochondrial activation followed a nucleated pattern, with specific, activation starting points at the midpiece from which mitochondrial activation was spread. Moreover, four separate sperm subpopulations were detected following the JC-1 orange$\mathrm{red} / \mathrm{green}$ ratio, and the observed changes in the mean JC-1 staining during IVC and IVAR were related to concomitant changes in both the orange-red/green JC-1 ratio and the percentage of sperm included in each subpopulation. All of these results indicate that IVC and the first minutes of IVAR are accompanied by a progressive increase in mitochondrial activity, which reached a peak coincidental with the achievement of IVAR. Moreover, results suggest the presence of separate sperm subpopulations, which show a different mitochondrial sensitivity to IVC and IVAR. Finally, mitochondrial activation, at least under JC-1 staining, seems to originate in concrete nucleation points at the midpiece, thus suggesting thus a well-coordinated pattern in boar-sperm mitochondrial activity modulation.

\section{Introduction}

Capacitation has been defined as the overall changes that the sperm undergoes after being ejaculated that allow it to fertilize the oocyte (Austin 1951; Chang 1951; Yanagimachi 1994). Capacitation results in altered plasma membrane architecture and permeability, which ultimately modulates flagellar activity and renders the sperm apical-head plasma membrane fusogenic (Rodríguez-Martínez 2007). Physiologically, spermatozoa acquire fertilization-competence in the female reproductive tract, but 'in vitro' capacitation (IVC) should also be achieved in defined media, the composition of which approximates the environment of the female reproductive tract (Visconti and Kopf 1998).

'In vitro' capacitation induced several sperm intracellular changes, such as increases in membrane fluidity, cholesterol efflux, intracellular $\mathrm{Ca}^{2+}$ and cAMP concentrations, protein tyrosine phosphorylation and variations in swimming patterns and chemotactic motility which are known as hyperactivated motility (Breitbart and Naor 1999). Despite this, little is known regarding the energy sources from which sperm carries out these transformations. Regarding motility, the source of ATP that supports this function has long been debated in the field of gamete research. In mammalian sperm, there are two pathways for ATP production: glycolysis, which occurs along the entire length of the principal piece of the flagellum, and mitochondrial respiration, centred on mitochondria of the midpiece. Mitochondrial respiration is the most efficient source of ATP and, in this way, it has been inferred that, under normal conditions, the ATP required for sperm motility is mainly obtained through mitochondrial respiration (Mukai and Okuno 2004). Hence, mitochondrial status has been related not only to sperm motility in bull (Garner and Thomas 1999), horse (Gravance et al. 2000), ram (MartínezPastor et al. 2004) and mouse (Mukai and Okuno, 2004) but also to fertilization ability in human (Kasai et al. 2002). However, several works strongly indicate that mitochondria are not the only energy source for sperm motility. In this way, the gene knock-out of the glycolytic enzyme glyceraldehyde 3-phosphate dehydrogenase (GAPDH), which catalyses the conversion of glyceraldehyde 3-phosphate to glycerate 1,3-bisphosphate in the glycolytic pathway, caused the appearance of non-motile sperm and a significant reduction in the ATP content $(10 \%$ of the total) despite having no deficiency in oxygen consumption (Miki et al. 2004). Accordingly, Marin et al. (2003) reported that glycolysis plays a significant role as an energy source in boar sperm. This is in accordance with the presence of an active and specific glycolytic activity in mammalian sperm (Mukai and Okuno 2004). This will be linked to the presence of a sperm-specific form of GAPDH, which is tightly bound to the fibrous sheath (Kamp et al. 2003). In addition, several studies have documented the relationship between glycolysis and capacitation-dependent cell signalling (Urner and Sakkas 2003). Following all of these results, Guthrie et al. (2008) propose glycolysis as a specialized and sperm-specific source of ATP to maintain motility in mammalian sperm. All of 
these data suggest that the maintenance of sperm function would be the result of the equilibrium between the energy obtained from glycolysis and the Krebs cycle.

The main aim of this work is to describe the changes in mitochondrial activity which are concomitant to IVC and subsequent 'in vitro', progesterone-induced acrosome reaction (IVAR) in boar spermatozoa. For this purpose, IVC- and IVAR-linked changes in mitochondrial activity through two separate techniques were analysed. The first one was the quantification of changes in the rhythm of oxygen consumption. The second one was the study of mitochondrial activity through the JC-1 staining procedure, analysing this through quantification of JC-1 intensity in digitalized, confocal laser microscope images.

\section{Materials and Methods}

\section{Boar semen collection}

All procedures described within were approved by the Autonomous University of Barcelona Animal Care and Use Committee and were performed in accordance with the Animal Welfare Law issued by the Catalan Government (Generalitat de Catalunya, Spain).

Commercial artificial insemination (AI) doses from boars of proven fertility were obtained from a commercial farm (Servicios Genéticos Porcinos, S.L.; Roda de Ter, Spain) The rich sperm fraction of the ejaculate was manually collected, maintained at $37^{\circ} \mathrm{C}$ in a water bath for a maximum of $2-3 \mathrm{~min}$ and diluted at $2 \times 10^{7}$ sperm $/ \mathrm{ml}$ in a commercial dose extender for refrigerated semen (MR-A Extender; Kubus, S.A.; Majadahonda, Spain) and distributed in 100-ml commercial doses. Six of the 100-ml doses obtained, chosen at random, coming from different boars, were placed in a portable refrigerator at $16^{\circ} \mathrm{C}$ for approximately $45 \mathrm{~min}$, which was the time required to arrive at the laboratory as previously reported (Ramió-Lluch et al. 2009).

\section{'In vitro' capacitation and acrosome reaction procedures}

Fifty microlitres of each AI dose was washed three times by centrifugation at $600 \mathrm{~g}$ for $5 \mathrm{~min}$ at $16^{\circ} \mathrm{C}$ and rediluted in a $20 \mathrm{~mm}$ 4-(2-hydroxyethyl)-1-piperazineethanesulfonic acid (Hepes) buffer (pH 7.4), containing $112 \mathrm{~mm}$ $\mathrm{NaCl}, 3.1 \mathrm{~mm} \mathrm{KCl}, 5 \mathrm{~mm}$ glucose, $21.7 \mathrm{~mm}$ L-lactate, $1 \mathrm{~mm}$ sodium pyruvate, $0.3 \mathrm{~mm} \mathrm{Na} \mathrm{NaPO}_{4}, 0.4 \mathrm{~mm}$ $\mathrm{MgSO}_{4}$ and $4.5 \mathrm{~mm} \mathrm{CaCl} 2$ [non-capacitating medium (NCM)]. The osmolarity was $304 \pm 5 \mathrm{mOsm}$, and $\mathrm{pH}$ was adjusted to 7.4. After the last wash, the sperm was resuspended in capacitating medium [Capacitation medium (CM) which is composed of NCM medium with $5 \mathrm{mg} / \mathrm{ml}$ bovine serum albumin (BSA) added] to a final concentration of $50-70 \times 10^{6} \mathrm{sperm} / \mathrm{ml}$. Incubation in $\mathrm{CM}$ was maintained for $4 \mathrm{~h}$ at $38.5^{\circ} \mathrm{C}$ in a $5 \% \mathrm{CO}_{2}$ atmosphere as described by Ramió et al. (2008).

The induction of IVAR was carried out through incubation in the presence of progesterone, as described before (Jiménez et al. 2003; Wu et al. 2006; Ramió et al. 2008). For this purpose, progesterone (Catalog Number P0130; stock concentration of $40 \mathrm{mg} / \mathrm{ml}$ in ethanol; Sigma, St. Louis, MO, USA) was added to a final concentration of $10 \mu \mathrm{g} / \mathrm{ml}$ with boar sperm previously incubated in $\mathrm{CM}$ for $4 \mathrm{~h}$ at $38.5^{\circ} \mathrm{C}$ in a $5 \% \mathrm{CO}_{2}$ atmosphere. After mixing thoroughly, the sperm was further incubated for an additional $1 \mathrm{~h}$ at $38.5^{\circ} \mathrm{C}$ in a $5 \% \mathrm{CO}_{2}$ atmosphere.

Sperm aliquots of $1.5 \mathrm{ml}$ were taken at $0,1,2,3$ and $4 \mathrm{~h}$ of capacitation and 5, 15, 30 and $60 \mathrm{~min}$ after the induction of IVAR. For SDS-PAGE and subsequent western blot analysis of tyrosine phosphorylation, sperm pellets were obtained by centrifugation $(30 \mathrm{~s}$ at $10000 \mathrm{~g}$ ) and immediately frozen at $-196^{\circ} \mathrm{C}$ in liquid $\mathrm{N}_{2}$ and stored at $-80^{\circ} \mathrm{C}$ before use.

\section{Evaluation of the achievement of capacitation status}

The evaluation of both IVC and IVAR was performed as described in Ramió et al. (2008). In this way, the parameters utilized to test the achievement of both IVC and IVAR were the percentage of viable sperm subjected to acrosome reaction (true acrosome reaction), the mean values of motility parameters after a computer-assisted analysis system (CASA) and the appearance of a specific tyrosine-phosphorylated protein with an estimated molecular weight of approximately $32 \mathrm{kDa}$.

The percentage of true acrosome reaction was analysed through the simultaneous estimation of both the percentages of viability and altered acrosomes by applying the following stains according to Bussalleu et al. (2005). In this technique, an aliquot of sperm suspension is first incubated with a stock solution of $15 \mathrm{~mm}$ bis-benzamidine to a final proportion of $1: 1000$ (v/v; bis-benzamidine/sperm suspension) for $10 \mathrm{~min}$ at $37^{\circ} \mathrm{C}$. Afterwards, a $2 \mathrm{~mm}$ propidium iodide stock solution is added to a final proportion of $6: 1000$ ( $\mathrm{v} / \mathrm{v}$; propidium iodide/sperm suspension), and the sperm is subjected to further incubation for $10 \mathrm{~min}$ at $37^{\circ} \mathrm{C}$. After this incubation, the sperm suspension is centrifuged at $1500 \mathrm{~g}$ for $10 \mathrm{~min}$, and the supernatant is discarded. The sperm pellet obtained is resuspended in $1 \mathrm{ml}$ of a solution of a final concentration of $100 \mathrm{~nm}$ Mitotracker ${ }^{\circledR}$ Green FM SBTI (Molecular Probes, Eugene, OR, USA, concentration of the stock solution: $1 \mathrm{~mm}$ ) and $100 \mathrm{~nm}$ FM-Alexa Fluor ${ }^{\circledR}$ 488-conjugated SBTI (Molecular probes, concentration of the stock solution: $1 \mathrm{~mm}$ ) in $\mathrm{CM}$ without BSA. The sperm suspension was incubated in this solution for $20 \mathrm{~min}$ at $37^{\circ} \mathrm{C}$ and was then immediately centrifuged at $1500 \mathrm{~g}$ for $12 \mathrm{~min}$. The resultant supernatant was discarded, whereas the sperm pellet was again resuspended in $100 \mathrm{ml}$ of $\mathrm{CM}$ without $\mathrm{BSA}$ at $37^{\circ} \mathrm{C}$. The sperm suspension was spread onto slides, and fluorescence was immediately determined in a Zeiss Axioskop-40 fluorescence microscope (Carl Zeiss GmbH, Jena, Germany) with the appropriate filters. Viability and altered acrosome percentages were determined after counting 200-300 spermatozoa per slide at a magnification of $1000 \times$. Unaltered acrosomes were considered to be those which showed a faint-to-moderate and uniform STBI lectin stain, whereas altered acrosomes showed a very faint and non-uniform stain. Sperm subjected to a true acrosome reaction were considered to be those which showed, after the stimulation of IVAR, positive 
viability (blue stain of the sperm head) and an intense and non-uniform STBI lectin stain. Finally, non-viable sperm showed an intense red stain of the head.

The CASA analysis was performed using a commercial system (Integrated Sperm Analysis System V1.0; Proiser, Valencia, Spain). In this procedure, samples were previously warmed at $37^{\circ} \mathrm{C}$ for $5 \mathrm{~min}$ in a water bath, and 5-ml aliquots of these samples were then placed onto a warmed $\left(37^{\circ} \mathrm{C}\right)$ slide and covered with a $22 \times 22 \mathrm{~mm}$ coverslip. Our CASA system was based upon the analysis of 25 consecutive, digitalized photographic images obtained from a single field at a magnification of $100 \times$ in a positive phase-contrast microscope at a capture velocity of 25 pictures/s. The sperm motility descriptors obtained were described following Ramió et al. (2008). Motility descriptors obtained after CASA analysis were:

Curvilinear velocity (VCL): the mean path velocity of the sperm head along its actual trajectory $(\mu \mathrm{m} / \mathrm{s})$.

Linear velocity (VSL): the mean path velocity of the sperm head along a straight line from its first to its last position $(\mu \mathrm{m} / \mathrm{s})$.

Mean velocity (VAP): the mean velocity of the sperm head along its average trajectory $(\mu \mathrm{m} / \mathrm{s})$.

Linearity coefficient (LIN): (VSL/VCL) $\times 100(\%)$.

Straightness coefficient (STR): (VSL/VAP) $\times 100(\%)$.

Wobble coefficient (WOB): (VAP/VCL) $\times 100(\%)$.

Mean amplitude of lateral head displacement (ALH): the mean value of the extreme side-to-side movement of the sperm head in each beat cycle $(\mu \mathrm{m})$.

Frequency of head displacement (BCF): the frequency with which the actual sperm trajectory crosses the average path trajectory $(\mathrm{Hz})$.

Finally, total motility was defined as the percentage of spermatozoa which showed a VAP above $10 \mu \mathrm{m} / \mathrm{s}$.

The determination of the tyrosine phosphorylation (Tyr-Phos) patterns was performed through a western blot analysis (Ramió et al. 2008). For this purpose, sperm samples were homogenized by sonication in icecold $10 \mathrm{~mm}$ Tris- $\mathrm{HCl}$ buffer $(\mathrm{pH}$ 7.4) containing $1 \%$ $(\mathrm{w} / \mathrm{v})$ sodium dodecyl sulphate (SDS) and $1 \mathrm{~mm}$ $\mathrm{Na}_{2} \mathrm{VO}_{4}$ (proportion $1: 5, \mathrm{v} / \mathrm{v}$ ) to avoid changes in the overall phosphorylation of the homogenates. Samples were briefly boiled and were then centrifuged at $10000 \mathrm{~g}$ for $15 \mathrm{~min}$ at $4^{\circ} \mathrm{C}$. Western blot analyses were performed on the supernatants obtained after this centrifugation. The analysis was based on SDS gel electrophoresis (Laemmli 1970) followed by transfer to nitrocellulose (Burnette 1981). The transferred samples were tested with an antiphosphotyrosine antibody (PY-20; Chemicon International; Temecula, CA, USA) at a dilution of $1: 1000(\mathrm{v} / \mathrm{v})$. Immunoreactive proteins were tested using peroxidase-conjugated anti-rabbit secondary antibody (Amersham, Buckinghamshire, UK), and the reaction was developed with an ECL-Plus detection system (Amersham). As previously described (Tardif et al. 2001; Dubé et al. 2005), IVC in boar sperm is associated with a specific increase in pTyr of a $32-\mathrm{kDa}$ protein, which was identified as proacrosin-binding protein 5. In this way, the appearance of this band was taken as a specific signal of the achievement of IVC. Thus, only those experiments in which a Tyr-Phos band of approximately $32 \mathrm{kDa}$ was detected were utilized for this work (See Fig. 1 as an example).

\section{Oxygen consumption measurement}

Sperm oxygen consumption was estimated with a Clark oxygen electrode linked to recorder system software (Oxygraph; Hansatech Instruments Ltd., Norfolk, UK), as in McLean et al. (1993). Water maintained at $38.5^{\circ} \mathrm{C}$ was circulated throughout the DW1 oxygen electrode chamber and constant stirring by a magnetic flea ensured homogeneous distribution of $\mathrm{O}_{2}$. The zero point was set by adding a few grains of sodium dithionite $\left(\mathrm{Na}_{2} \mathrm{~S}_{2} \mathrm{O}_{4}\right)$ to the chamber, which contained $700 \mu \mathrm{l}$ of distilled water. Measurements were taken by adding $900 \mu \mathrm{l} \mathrm{CM}$ heated to $38.5^{\circ} \mathrm{C}$ followed by $100 \mu \mathrm{l}$ of the sperm solution. The plunger was inserted to expel air, and $\mathrm{O}_{2}$ consumption was monitored for $3 \mathrm{~min}$. The mean sperm concentration at the DW1 chamber was $8 \times 10^{6} \mathrm{sperm} / \mathrm{ml}$. Data are presented as nmol $\mathrm{O}_{2}$ consumed $/ \mathrm{min} / 10^{7}$ viable sperm

\section{Evaluation of mitochondrial membrane through JC-1 staining}

The lipophilic cationic compound, 5,5',6,6'-tetrachloro$1,1^{\prime}, 3,3^{\prime}$-tetraethylbenzimidazolyl carbocyanine iodide (JC-1), has the distinct advantage of differentiating cells of high and low mitochondrial membrane potential. The maximal excitation for JC-1 is $488 \mathrm{~nm}$, with an emission spectrum of the monomer in the green range $(530 \mathrm{~nm})$ when mitochondria are inactive, and the emission of JC-1 aggregates in the high-orange wavelength $(590 \mathrm{~nm})$ when mitochondrial transmembrane potential (MMP) is high. Huo et al. (2002) have described the validity of this probe for boar sperm. A 1.5-ml aliquot of sperm was incubated with $15 \mu \mathrm{l}$ of JC-1 staining $(153 \mu \mathrm{M}$; T-3168; Molecular Probes, Eugene, OR, USA) for $10 \mathrm{~min}$ in the dark at $37^{\circ} \mathrm{C}$. The sperm was placed on clean microscope slides with a coverslip on the top. Images of the live stained

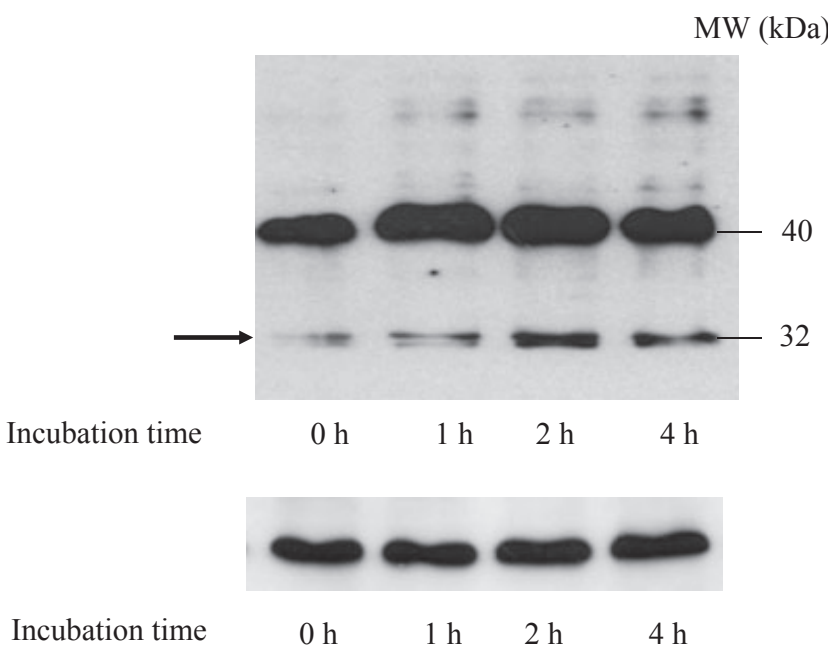

Fig. 1. Protein tyrosine phosphorylation pattern of boar sperm after 'in vitro' capacitation and subsequent 'in vitro' acrosome reaction revealed through western blot. The figure shows a representative image for all of the analysed experiments. Arrow indicate the position of the phosphorylated, capacitation-specific, p32 protein 
sperm were obtained with a Leica TCS 4D confocal scanning microscope (Leica Lasertechnik GmbH; Heidelberg, Germany) adapted to an inverted Leitz DMIRBE microscope and a $63 \times$ (NA 1.4 oil) Leitz Plan-Apo lens (Leitz; Stuttgart, Germany) in a $5 \% \mathrm{CO}_{2}$ atmosphere and with a thermoplate at $38.5^{\circ} \mathrm{C}$. The light source was an argon/krypton laser. Images and videos were subsequently stored as TIFF-format images and AVI format videos, respectively. Images were analysed by computerized analysis in the Adobe Photoshop 6.0 image processor program (Adobe Systems; Mountain View, CA, USA). A minimum of 30 spermatozoa per sample was scored. A total of six experiments were performed. Each spermatozoon was analysed individually; for this purpose, the analysed midpieces were individually delimited. After this, both green- and red/orange-colour intensities of these delimited midpieces were calculated using the 'Histogram' included in the program. The values obtained after the application of this procedure were further processed to obtain two separate intensity scales following mean intensity values per pixel, one for each colour, with arbitrary values ranging from 1 (minimal intensity) to 256 (maximal intensity). Afterwards, the proportion of colour intensities between orange and green was individually calculated for each midpiece, and a further statistical analysis was performed to yield the results described as follows (See the Statistics).

\section{Statistics}

Statistical analysis of the data obtained with the JC-1 staining procedure was performed by applying the SAS statistical package (SAS Statistical Analysis System 1982). As described earlier, the analysed variable was the proportion between orange colour intensity and green colour intensity. A previous PROC NORM PLOT procedure was performed to determine that the obtained data distribution did not follow a normal distribution. Afterwards, data were normalized by applying a logarithmic data transformation. A FASTCLUS procedure was then applied to allocate every individual spermatozoon into a specific sperm subpopulation. The FASTCLUS procedure performs a disjointed cluster analysis based on Euclidean distances computed from one or more quantitative parameter variables. Spermatozoa were divided into clusters, such that every observation belonged to a single cluster. Sperm cells that shared similar fluorescence were assigned to the same cluster, whereas spermatozoa that differed were assigned to different clusters. A PROC GLM procedure was applied to evaluate significant differences $(\mathrm{p}<0.05)$, and the LSMEANS procedure was applied to list these differences. Finally, a Chi-square procedure was applied to determine the subpopulation distribution percentage of every single experiment. Once the percentage distribution per experiment was determined, new PROC GLM and LSMEANS procedures were applied to determine and list, respectively, the differences among the different time points of IVC and IVAR procedures. The total number of spermatozoa analysed following this protocol was 800 . These cells were from a total of six separate experiments, thus indicating that the analysis was performed on 100-150 sperm per sample. Finally, PROC GLM and LSMEANS procedures were also applied to test significant differences in the results concerning the rhythm of $\mathrm{O}_{2}$ consumption.

\section{Suppliers}

All of the supplies were of analytical grade and came from Sigma, Boehringer-Mannheim (Mannheim, Germany) and Merck (Darmstadt, Germany).

\section{Results}

\section{Evaluation of capacitation status during IVC and IVAR}

The induction of IVC induced a progressive decline in the percentage of viability, which was concomitant to an increase in the percentage of altered acrosomes (Table 1).

Table 1. Percentages of viability, altered acrosomes, true acrosome reaction and mean motility parameters of boar sperm subjected to 'in vitro' capacitation and subsequent 'in vitro' progesterone-induced acrosome reaction

\begin{tabular}{|c|c|c|c|c|c|c|c|c|c|c|}
\hline \multirow[b]{2}{*}{ Incubation time } & \multicolumn{6}{|c|}{ Incubation in capacitating medium } & \multicolumn{4}{|c|}{ Time after progesterone addition } \\
\hline & $0 \mathrm{~h}$ & & $1 \mathrm{~h}$ & $2 \mathrm{~h}$ & $3 \mathrm{~h}$ & $4 \mathrm{~h}$ & $5 \mathrm{~min}$ & $15 \mathrm{~min}$ & $30 \mathrm{~min}$ & $60 \mathrm{~min}$ \\
\hline Viability (\%) & $78.0 \pm 4.2^{\mathrm{a}}$ & 70.9 & $\pm 4.5^{\mathrm{a}}$ & $69.0 \pm 3.2^{\mathrm{a}}$ & $70.2 \pm 4.5^{\mathrm{a}}$ & $69.5 \pm 2.2^{\mathrm{a}}$ & $67.0 \pm 2.6^{\mathrm{ab}}$ & $66.4 \pm 2.6^{\mathrm{ab}}$ & $64.7 \pm 2.6^{\mathrm{ab}}$ & $62.0 \pm 2.7^{\mathrm{b}}$ \\
\hline $\begin{array}{l}\text { Total altered } \\
\text { acrosomes }(\%)\end{array}$ & $12.3 \pm 5.9^{\mathrm{a}}$ & 26.0 & $\pm 3.1^{\mathrm{b}}$ & $31.7 \pm 3.2^{\mathrm{b}}$ & $30.9 \pm 3.1^{\mathrm{b}}$ & $29.4 \pm 4.0^{\mathrm{b}}$ & $38.8 \pm 4.8^{\mathrm{b}}$ & $52.7 \pm 4.0^{\mathrm{c}}$ & $64.0 \pm 3.4^{\mathrm{d}}$ & $64.2 \pm 4.0^{\mathrm{d}}$ \\
\hline $\begin{array}{l}\text { True acrosome } \\
\text { reaction }(\%)\end{array}$ & $2.2 \pm 1.8^{\mathrm{a}}$ & 6.1 & $\pm 2.1^{\mathrm{a}}$ & $11.5 \pm 3.0^{\mathrm{ab}}$ & $10.9 \pm 2.8^{\mathrm{ab}}$ & $9.4 \pm 2.2^{\mathrm{ab}}$ & $29.0 \pm 3.8^{\mathrm{b}}$ & $33.5 \pm 4.3^{\mathrm{b}}$ & $40.5 \pm 2.9^{\mathrm{bc}}$ & $44.0 \pm 4.7^{\mathrm{c}}$ \\
\hline Total motility $(\%)$ & $79.8 \pm 1.1^{\mathrm{a}}$ & 77.9 & $\pm 1.4^{\mathrm{a}}$ & $73.6 \pm 2.1^{\mathrm{ab}}$ & $71.0 \pm 1.1^{\mathrm{b}}$ & $69.0 \pm 2.0^{\mathrm{b}}$ & $70.2 \pm 1.5^{\mathrm{b}}$ & $67.8 \pm 1.5^{\mathrm{bc}}$ & $62.4 \pm 1.3^{\mathrm{c}}$ & $55.2 \pm 1.1^{\mathrm{d}}$ \\
\hline $\operatorname{VCL}(\mu \mathrm{m} / \mathrm{s})$ & $87.8 \pm 1.0^{\mathrm{a}}$ & 87.7 & $\pm 1.0^{\mathrm{a}}$ & $87.5 \pm 1.1^{\mathrm{a}}$ & $91.4 \pm 1.3^{\mathrm{a}}$ & $99.7 \pm 1.2^{\mathrm{b}}$ & $102.8 \pm 1.4^{\mathrm{b}}$ & $107.4 \pm 1.6^{\mathrm{b}}$ & $106.3 \pm 1.0^{\mathrm{b}}$ & $107.3 \pm 1.4^{\mathrm{b}}$ \\
\hline $\operatorname{VSL}(\mu \mathrm{m} / \mathrm{s})$ & $35.4 \pm 2.6^{\mathrm{a}}$ & 40.2 & $\pm 2.9^{\mathrm{a}}$ & $44.1 \pm 1.4^{\mathrm{b}}$ & $47.2 \pm 3.0^{\mathrm{bc}}$ & $51.3 \pm 1.8^{\mathrm{c}}$ & $53.0 \pm 2.6^{\mathrm{c}}$ & $56.3 \pm 1.9^{\mathrm{c}}$ & $53.2 \pm 1.9^{\mathrm{c}}$ & $54.0 \pm .1^{\mathrm{c}}$ \\
\hline $\operatorname{VAP}(\mu \mathrm{m} / \mathrm{s})$ & $48.5 \pm 2.3^{\mathrm{a}}$ & 52.0 & $\pm 2.6^{\mathrm{a}}$ & $58.3 \pm 2.5^{\mathrm{ab}}$ & $60.2 \pm 2.6^{\mathrm{b}}$ & $63.0 \pm 2.7^{\mathrm{b}}$ & $64.1 \pm 2.9^{\mathrm{b}}$ & $67.3 \pm 3.2^{\mathrm{b}}$ & $66.2 \pm 2.0^{\mathrm{b}}$ & $66.3 \pm 2.1^{\mathrm{b}}$ \\
\hline LIN $(\%)$ & $24.2 \pm 2.5^{\mathrm{a}}$ & 39.9 & $\pm 2.7^{\mathrm{ab}}$ & $47.1 \pm 2.9^{\mathrm{b}}$ & $47.3 \pm 3.0^{\mathrm{b}}$ & $47.9 \pm 2.8^{b}$ & $47.8 \pm 2.7^{\mathrm{b}}$ & $49.9 \pm 2.8^{\mathrm{b}}$ & $51.3 \pm 2.9^{\mathrm{b}}$ & $50.0 \pm 3.2^{\mathrm{b}}$ \\
\hline STR (\%) & $62.8 \pm 2.3^{\mathrm{a}}$ & 69.9 & $\pm 2.8^{\mathrm{ab}}$ & $74.3 \pm 2.5^{\mathrm{b}}$ & $73.1 \pm 2.0^{\mathrm{b}}$ & $75.3 \pm 2.7^{\mathrm{b}}$ & $76.5 \pm 2.4^{\mathrm{bc}}$ & $81.2 \pm 2.8^{\mathrm{c}}$ & $80.4 \pm 2.9^{\mathrm{c}}$ & $81.3 \pm 2.9^{\mathrm{c}}$ \\
\hline WOB $(\%)$ & $52.5 \pm 2.8^{\mathrm{a}}$ & 59.3 & $\pm 2.7^{\mathrm{ab}}$ & $64.9 \pm 2.9^{b}$ & $64.0 \pm 3.0^{\mathrm{b}}$ & $63.7 \pm 2.8^{b}$ & $63.0 \pm 2.7^{\mathrm{b}}$ & $62.5 \pm 2.6^{\mathrm{b}}$ & $62.1 \pm 2.9^{\mathrm{b}}$ & $62.2 \pm 2.7^{\mathrm{b}}$ \\
\hline Mean ALH $(\mu \mathrm{m})$ & $4.27 \pm 0.18^{\mathrm{a}}$ & 4.01 & $\pm 0.13^{\mathrm{a}}$ & $3.89 \pm 0.16^{\mathrm{ab}}$ & $3.80 \pm 0.19^{\mathrm{ab}}$ & $3.96 \pm 0.11^{\mathrm{ab}}$ & $4.35 \pm 0.17^{\mathrm{b}}$ & $4.47 \pm 0.18^{\mathrm{b}}$ & $4.43 \pm 0.15^{\mathrm{b}}$ & $4.40 \pm 0.13$ \\
\hline $\mathrm{BCF}(\mathrm{Hz})$ & $6.4 \pm 0.4^{\mathrm{a}}$ & 6.3 & $\pm 0.3^{\mathrm{a}}$ & $6.6 \pm 0.4^{\mathrm{a}}$ & $6.6 \pm 0.5^{\mathrm{a}}$ & $6.8 \pm 0.4^{\mathrm{a}}$ & $6.7 \pm 0.5^{\mathrm{a}}$ & $6.9 \pm 0.5^{\mathrm{a}}$ & $7.0 \pm 0.6^{\mathrm{a}}$ & $6.9 \pm 0.4^{\mathrm{a}}$ \\
\hline
\end{tabular}

ALH, amplitude of lateral head displacement; BCF, frequency of head displacement; LIN, linearity coefficient; STR, straightness coefficient; WOB, Wobble coefficient; VAP, mean velocity; VCL, curvilinear velocity; VSL, linear velocity.

Semen quality parameters have been defined in the Materials and Methods. Results are expressed as mean \pm SEM for 16 separate experiments. Different superscripts in a row indicate significant $(\mathrm{p}<0.05)$ differences among groups after a PROC GLM of the SAS statistical package. 
The analysis of the true acrosome reaction indicated that this percentage was very low at $0 \mathrm{~h}$ of incubation in the capacitation medium $(2.2 \pm 1.8 \%$, mean \pm SEM $)$, and there was only a slight increase after $4 \mathrm{~h}$ of incubation (Table 1). Moreover, a progressive decrease in total motility, which was parallel to that observed in viability, was detected. Notwithstanding, the mean motility characteristics of motile sperm indicated a progressive and significant $(\mathrm{p}<0.05)$ increase in VCL, VSL, VAP, LIN, STR and WOB after $4 \mathrm{~h}$ of incubation (Table 1). The subsequent induction of IVAR caused a further and great increase in the percentage of true acrosome reaction, which reached values from $29.0 \pm 3.8 \%$ after 5 min of IVAR induction to $44.0 \pm 3.7 \%$ after $60 \mathrm{~min}$ of progesterone addition (Table 1). This was not accompanied by any significant change in the mean motility patterns, which maintained the values observed after the attainment of IVC (Table 1). As indicated previously, in all of the analysed experiments, the appearance of a specific, 32-kDa tyrosine-phosphorylated protein was mandatory and concomitant with the changes described (Fig. 1 as an example, and data not shown).

\section{Effects of IVC and IVAR on the rhythm of oxygen consumption}

Incubation of boar sperm in the CM induced a slight and progressive, time-dependent increase in the rhythm of oxygen consumption. This increase reached a maximum after 3-4 h of incubation (from $1.61 \pm 0.08 \mathrm{nmol}$ $\mathrm{O}_{2} / \mathrm{min} / 10^{7}$ viable sperm at $0 \mathrm{~h}$ to $1.97 \pm 0.08 \mathrm{nmol}$ $\mathrm{O}_{2} / \mathrm{min} / 10^{7}$ viable sperm after $4 \mathrm{~h}$ of incubation. See Fig. 2). The induction of IVAR caused a rapid and significant $(\mathrm{p}<0.05)$ increase in oxygen consumption, which reached values of $2.62 \pm 0.12 \mathrm{nmol} \mathrm{O} \mathrm{O}_{2} / \mathrm{min} / 10^{7}$ viable sperm after $5 \mathrm{~min}$ of progesterone addition. Afterwards, a time-dependent decrease in oxygen consumption was observed, attaining values of $1.06 \pm 0.05$ nmol $\mathrm{O}_{2} / \mathrm{min} / 10^{7}$ viable sperm after $60 \mathrm{~min}$ of the IVAR induction (Fig. 2).

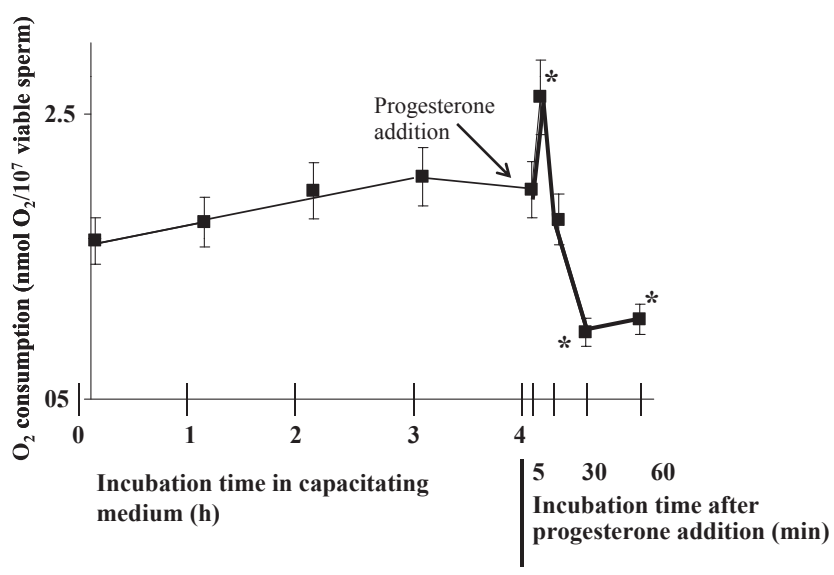

Fig. 2. Oxygen consumption during 'in vitro' capacitation (IVC) and subsequent, progesterone-induced 'in vitro' acrosome reaction (IVAR). Boar sperm was subjected to IVC through incubation for $4 \mathrm{~h}$ in the $\mathrm{CM}$ and was then further subjected to progesterone-stimulated IVAR (starting point marked with an arrow) as described in the Materials and Methods. Asterisks indicate the presence of significant $(\mathrm{p}<0.05)$ differences, when comparing the result to the 0 -h incubation point Results are expressed as mean \pm SEM for six separate experiments

\section{Effects of IVC and IVAR on the mitochondrial membrane} potential measured through $\mathrm{JC}-1$ staining

The microscopic study of the images obtained after JC-1 staining showed the existence of a specific pattern of sperm-midpiece mitochondrial activation. Thus, as shown in Fig. 3, if considering that green mitochondria have low MMP and red mitochondria have the most intense MMP, the appearance of the red stain started at small points, mainly located at the extremes of the midpiece, from which the red JC-1 staining extended until occupying the whole midpiece in the most activated sperm. This indicates an activation pattern based on punctual activation nuclei, from which the activating signal was dispersed to the other mitochondria of the midpiece.

Boar sperm showed a mean orange/green ratio of JC-1 staining of $8.2 \pm 0.1$ at $0 \mathrm{~h}$ of incubation in $\mathrm{CM}$ (mean $\pm \mathrm{SEM}$ ). This mean ratio did not significantly increase after incubation in the CM medium, reaching values of $8.6 \pm 0.2$ after $4 \mathrm{~h}$ of incubation (Table 2). The induction of IVAR did not induce any significant increase in the mean values of the orange/green ratio of JC-1 staining after $5 \mathrm{~min}$ of the progesterone addition either (Table 2). Finally, a decrease in the mean values of the JC-1 orange/green proportion was observed after $60 \mathrm{~min}$ of the progesterone addition ( $4.9 \pm 0.1$; see Table 2). On the other hand, JC-1 analysis rendered the presence of 4 separate sperm subpopulations in fresh semen, in accordance with their midpiece orange/green stain proportion. These subpopulations were the following:

Subpopulation 1: Included spermatozoa with the highest MMP activity. At $0 \mathrm{~h}, 27.7 \%$ of the total spermatozoa showed a mean 14.7-fold red/orange intensity when compared with the green colour.

Subpopulation 2: Spermatozoa with high MMP activity but lower than Subpopulation 1 (mean orange/red-green intensity proportion: 6.7). Approximately $37.3 \%$ of the sperm were classified in this subpopulation at $0 \mathrm{~h}$ of incubation and showed a clearly predominant orange fluorescence.

Subpopulation 3: Spermatozoa of this subpopulation showed a lower MMP when compared with Subpopulations 1 and 2 (mean orange/red-green proportion of approximately 3.0 ). At $0 \mathrm{~h}$ of incubation, $31.6 \%$ of the total sperm were included in this subpopulation.

Subpopulation 4: Spermatozoa included in this subpopulation showed the lowest MMP activity (mean orange/red-green proportion of approximately 0.8 ). At $0 \mathrm{~h}$ of incubation, Subpopulation 4 represented $3.7 \%$ of the overall sperm.

In contrast to mean values, the incubation of boar sperm in CM induced clear changes in both the orange/ green ratio and the proportion of cells included in each subpopulation. In this way, incubation for $4 \mathrm{~h}$ in the capacitating medium induced a significant $(\mathrm{p}<0.05)$ increase in the orange/green ratio in Subpopulations 1, 2 and 3, which reached values, in Subpopulation 1, from $14.7 \pm 1.3$ at $0 \mathrm{~h}$ of incubation to $47.5 \pm 1.5$ after $4 \mathrm{~h}$ (Table 2). The induction of acrosome reaction induced 
Subpopulations

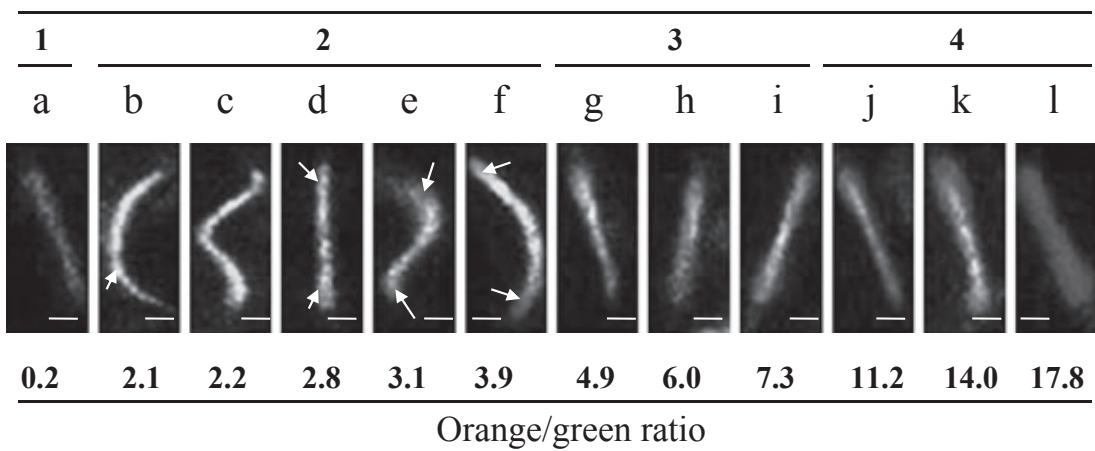

Fig. 3. Location changes in mitochondria with activity changes through JC-1 mitochondrial staining in boar spermatozoa. The figure shows the appearance of local focuses of active mitochondria (yellow-red areas) among non-active mitochondria (green areas) in boar sperm from low (a) to maximal mitochondrial activity (1). Arrows indicate the location of initial points of mitochondrial activation in sperm with low overall mitochondrial activity. Bars indicate a real size of $2 \mu \mathrm{m}$. (a) Typical sperm from Subpopulation 1. (b-f) Sperm included in Subpopulation 2. (g-i) Sperm included in Subpopulation 3. (j-1) Sperm included in Subpopulation 4. The orange/green ratio scale indicates the precise orange/green ratio of each of the sperm shown

Table 2. Values of the orange/green JC-1 fluorescence ratio of the whole ejaculate and of the obtained subpopulations in boar sperm subjected to 'in vitro' capacitation and subsequent, progesteroneinduced 'in vitro' acrosome reaction

\begin{tabular}{lrrrrr}
\hline & \multicolumn{2}{c}{$\begin{array}{c}\text { Incubation time in the } \\
\text { capacitating medium (h) }\end{array}$} & & \multicolumn{2}{c}{$\begin{array}{c}\text { Time after progesterone } \\
\text { addition (min) }\end{array}$} \\
\cline { 2 - 3 } \cline { 5 - 6 } & 0 & 4 & & 5 & 60 \\
\hline $\begin{array}{l}\text { Whole ejaculate } \\
\text { (mean JC-1 ratio }\end{array}$ & $8.2 \pm 0.1^{\mathrm{a}}$ & $8.6 \pm 0.2^{\mathrm{a}}$ & & $8.3 \pm 0.1^{\mathrm{a}}$ & $4.9 \pm 0.1^{\mathrm{c}}$ \\
values) & & & & & \\
Subpopulation 1 & $14.7 \pm 1.3^{\mathrm{ab}}$ & $47.5 \pm 1.5^{\mathrm{c}}$ & & $15.8 \pm 1.4^{\mathrm{a}}$ & $11.9 \pm 1.5^{\mathrm{b}}$ \\
Subpopulation 2 & $6.7 \pm 1.2^{\mathrm{a}}$ & $11.5 \pm 1.3^{\mathrm{b}}$ & $6.9 \pm 1.3^{\mathrm{a}}$ & $3.9 \pm 1.3^{\mathrm{c}}$ \\
Subpopulation 3 & $3.0 \pm 1.3^{\mathrm{a}}$ & $5.0 \pm 1.3^{\mathrm{b}}$ & $3.0 \pm 1.3^{\mathrm{a}}$ & $1.6 \pm 1.4^{\mathrm{c}}$ \\
Subpopulation 4 & $0.8 \pm 1.5^{\mathrm{a}}$ & $1.1 \pm 1.3^{\mathrm{a}}$ & $1.6 \pm 1.4^{\mathrm{b}}$ & $0.2 \pm 1.6^{\mathrm{c}}$ \\
\hline
\end{tabular}

Results are mean \pm SEM for five separate experiments. Different superscripts in a row indicate significant $(\mathrm{p}<0.05)$ differences among groups. The obtainment of subpopulations and the orange/green JC-1 ratio is described in the corresponding Materials and Methods. Furthermore, values are expressed as arbitrary units, as also described in the Materials and Methods.

a decrease in the orange/green ratio in all subpopulations also, attaining values, in Subpopulation 1, of $11.9 \pm 1.5$ after 60 min of progesterone addition (Table 2).

Regarding sperm distribution among subpopulations, the percentage of sperm included in Subpopulation 1 progressively decreased, going from $27.7 \%$ at $0 \mathrm{~h}$ of incubation to $7.2 \%$ after $4 \mathrm{~h}$ of incubation (Fig. 4). This decrease was concomitant with a moderate increase in Subpopulations 2 (from $37.3 \%$ at $0 \mathrm{~h}$ of incubation to $45.2 \%$ after $4 \mathrm{~h}$ of incubation) and 3 (from $31.7 \%$ at $0 \mathrm{~h}$ of incubation to $41.6 \%$ after $4 \mathrm{~h}$ of incubation; see Fig. 4). The induction of IVAR caused, on the contrary, a very rapid increase in the proportion of sperm included in Subpopulation 1, which reached values of $39.0 \% 5 \mathrm{~min}$ after of the addition of progesterone. Likewise, sperm in Subpopulation 4 also increased, going from $5.9 \%$ at the moment of the progesterone addition to $19.4 \%$ after 5 min of incubation (Fig. 4). These increases were linked to concomitant decreases in sperm from Subpopulations 2 and 3. Afterwards, there

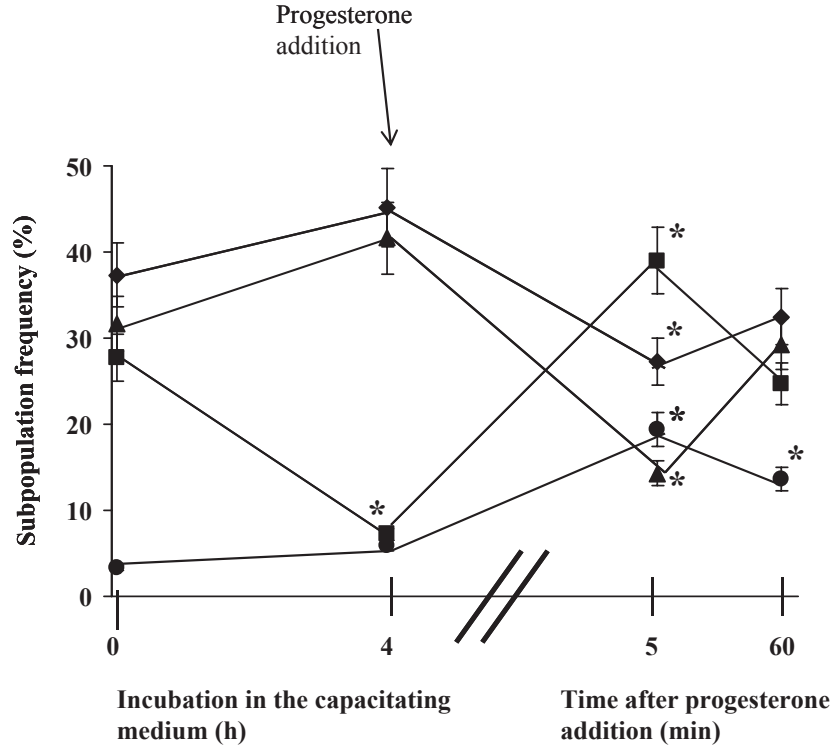

Fig. 4. Dynamics of the boar JC-1 sperm subpopulations structure during 'in vitro' capacitation (IVC) and further progesterone-induced 'in vitro' acrosome reaction (IVAR). JC-1 sperm subpopulations have been defined in the Materials and Methods. $\square$ : Subpopulation 1. Subpopulation 2. $\mathbf{\Delta}$ : Subpopulation 3. O: Subpopulation 4. Results shown are mean \pm SEM for five separate experiments. Asterisks indicate the presence of significant $(\mathrm{p}<0.05)$ differences, when comparing the result to the 0 - $\mathrm{h}$ incubation point. Subpopulation frequency must be interpreted as the percentage of spermatozoa that was included in the respective subpopulation. The horizontal scale is different for IVC and IVAR (starting point marked with an arrow) to better show the results after progesterone addition

were slight changes in the percentage of sperm included in each subpopulation, with moderate-to-slight decreases in Subpopulations $1(24.7 \%$ after $60 \mathrm{~min}$ of IVAR induction) and 4 (13.6\% after $60 \mathrm{~min}$ of progesterone addition) that were concomitant with moderateto-slight increases in Subpopulations $2(32.5 \%$ after $60 \mathrm{~min}$ of IVAR incubation) and $3(29.3 \%$ after $60 \mathrm{~min}$ of progesterone addition; see Fig. 4). 


\section{Discussion}

The results shown here highlighted a new perspective in the dynamics of mitochondrial function during the attainment of IVC and subsequent IVAR of boar sperm. This is sustained if it is considered that the results indicate three different aspects of the mitochondrial function. First, the interpretation of mean results indicates that IVC, and especially IVAR, activates separate mitochondrial functions, such as the complex Krebs cycle/electronic chain and the MMP-linked chemiosmosis with different intensities. Second, although mean values of JC-1 did not show changes in the overall MMP during IVC and AR, the analysis of the same results under a subpopulational point of view highlighted the achievement of important and specific changes linked to the sperm subpopulations after IVC and AR attainment. Third, our results also suggest that the increase in MMP is achieved through a nucleated, non-homogeneous, activation pattern in the wholesperm midpiece. These points are subsequently discussed in a more in-depth manner.

Regarding the appearance of a fast and intense peak of $\mathrm{O}_{2}$ consumption, but not of the MMP immediately after the progesterone-induced AR achievement, we must make several considerations. First, our results indicate that boar-sperm mitochondria have the ability to modulate two of the most important functions of these organelles in separate ways, namely the oxidative metabolism generated by the Krebs cycle and the linked electronic chain and the generation of high MMP (Rich 2003; McBride et al. 2006). The ability of boar sperm to separate the oxidative metabolism and MMP is in accordance with other previously published regarding mitochondrial function of boar sperm in conditions different to those described here, such as the incubation with fructose and storage (Aalbers et al. 1961) and incubation with a specific inhibitor of the glycerol 3-phosphate dehydrogenase (Jones and Gillan 1996). Furthermore, this ability is compatible with the interpretation of the results obtained in boar ejaculates when other sperm function characteristics are analysed. Thus, the observed changes in parameters like motility patterns (Ramió et al. 2008), plasma membrane fluidity (Harrison et al. 1996) and protein phosphorylation (Urner and Sakkas 2003) after IVC and IVAR are well explained through the observed different sensitivity of mitochondrial oxidative metabolism and MMP. In fact, our results suggest that the ultimate purpose of the oxidative metabolism and the MMP in boar sperm is different. In this way, it must be remembered that the achievement of IVC has been associated with the prevention of the activation of apoptotic transduction signals, at least in species like human (Mann et al. 2002; Grunewald et al. 2009) and ram (Martí et al. 2008). In a similar way, the production of reactive oxygen products (ROS) is related to the induction of the protein tyrosine phosphorylation that is concomitant with the achievement of capacitation (Travert et al. 2009). Remarkably, both the regulation of apoptotic transduction signals such as caspase 3 and caspase 9 activities and ROS generation are mainly regulated by mitochondrial activity. Specifically, the dissipation of MMP is associ- ated with the activation of the initial steps of apoptosis related to the activation of caspase 3 and caspase 9 (Krysko et al. 2001). Furthermore, the rhythm of ROS generation by mitochondria is inversely correlated with MMP, thus indicating a role of MMP in the control of mitochondrial ROS generation (Wang et al. 2003). All of these findings strongly suggest that the main role of MMP in boar sperm would not be the generation of ATP through chemiosmosis, but rather the control of other mitochondria-controlled processes that are important in the attainment of capacitation, like the activity of apoptotic-related pathways of the generation of ROS. This would be different for the oxidative metabolism represented in the Krebs cycle, which would be focused on the obtainment of energy needed for the achievement of IVC and IVAR. Thus, because the final objectives of the mitochondrial function analysed by $\mathrm{O}_{2}$ consumption and by $\mathrm{JC}-1$ staining are different, it is logical that their activation dynamics during IVC and IVAR would also be different. On the other hand, it is noteworthy that freshly obtained boar sperm only obtained approximately $5 \%$ of their energy through the Krebs cycle, whereas the main ATP source was glycolysis (Marin et al. 2003). If we compare the $\mathrm{O}_{2}$ consumption levels obtained in our experimental design with the same levels of cells with an active Krebs cycle, such as pig hepatocytes, we can observe that the total energy that boar sperm obtains from oxidative pathways is very low. Thus, whereas the maximal $\mathrm{O}_{2}$ rate observed in our study was $2.62 \mathrm{nmol} / \mathrm{min} / 10^{7}$ viable cells, the minimal rate described in pig hepatocytes was $18 \mathrm{nmol} / \mathrm{min} / 10^{6}$ viable cells (Balis et al. 1999). Taking into account that, in fact, the increase in $\mathrm{O}_{2}$ consumption generated by IVC and IVAR was, in fact, not very high, it can be assumed that glycolysis would continue to be the main ATP source during IVC and IVAR despite the $\mathrm{O}_{2}$ consumption burst observed after progesterone addition. In this way, the separate roles of the Krebs cycle/electronic chain as ATP generators and MMP as the regulatory mechanism of apoptotic transduction signals and ROS generation can be better understood.

Nonetheless, the analysis of the mean results does not reveal the whole picture. Analysis of JC-1 staining results under a subpopulational point of view reveals that IVC and subsequent IVAR induce significant changes in mitochondrial activity, at least in MMP. It is worth noting that, whereas the mean values of the orange/green $\mathrm{JC}-1$ ratio did not significantly change after $4 \mathrm{~h}$ of incubation in CM nor after $5 \mathrm{~min}$ of the progesterone addition, the specific subpopulational structure was totally different in each point. Thus, the attainment of IVC induces a great increase in the orange/green ratio of the most activated sperm, together with an increase in spermatozoa that showed mediumto-high orange/green JC-1 ratios. Furthermore, IVAR was concomitant with a quick increase in sperm with the highest orange/green JC-1 ratio. These results have several important features. The first would be the highlight of separate sperm populations with different sensitivities to IVC and IVAR, regarding their ability to increase MMP. These different sensitivities would be related to the presence of separate subpopulations in boar ejaculates that not only showed different motility 
characteristics but also changed their motility patterns in a different way when ejaculates were subjected to IVC and IVAR (Ramió et al. 2008). This leads one to suppose that sperm of freshly obtained boar ejaculates that showed specific features in functional aspects linked to their motion parameters and their MMP levels would have different sensitivities to achieve IVC and IVAR. A similar conclusion after observing other sperm function markers was achieved by Satake et al. (2006), who indicate that the interaction of boar sperm with oviductal proteins is related to the sensitivity of each spermatozoon to respond to bicarbonate stimulation, thus linking the specific sensitivity of each sperm when undergoing capacitation to its ability to reach oocytes. Unfortunately, at this moment we are not able to perform a simultaneous analysis of motility and MMP. This kind of analysis would test the feasibility of this hypothesis, but the possibility of the existence of sperm subpopulations with different abilities to achieve IVC and IVAR in boar ejaculates cannot be by any means dismissed.

Another important point regarding the subpopulational analysis of MMP is the ability to show changes in functions like MMP that were hidden when a mean analysis of data is performed. This conclusively indicates that the subpopulational analytical approach is much more sensitive to detect small and subtle changes in parameters like MMP than a mere analysis of mean data. In fact, the superiority of the subpopulational analysis as opposed to a mean analysis of data, especially when changes are small and subtle, has already been highlighted in other studies not directly linked to mitochondrial function. Thus, freezability of boar sperm has been related to subtle changes in both the specific values and the specific frequency of sperm included in the different subpopulations obtained in fresh boar ejaculates taking into account their motility parameters (Flores et al. 2009). All of this leads us to propose the subpopulational analysis approach as being the most feasible to yield the maximal information of any type of data regarding changes in sperm functionality.

Regarding results of $\mathrm{JC}-1$ staining, the putative existence of a specific pattern of mitochondrial activation along the midpiece is also important. Thus, our observations strongly suggest that mitochondrial activation does not start in a homogeneous pattern through the entire midpiece, but rather from concrete points, generally located at the extremes of the midpiece, from which mitochondrial activation is radiated out to the entire piece. This suggestion is based on a chronological analysis of the results obtained. Thus, the existence of sperm that shows the presence of nucleation activating points is observable in all of the incubation times, as there is sperm with different mitochondrial activation status in all of the studied points. Strikingly, all of the sperm that presents the nucleated pattern is all included in Subpopulation 2, whereas those included in Subpopulation 3 showed more widespread activation zones in the midpiece, and those of Subpopulation 4 showed a regular orange staining in all of the midpiece. In this way, the assumption of a nucleated activation pattern for MMP is based on the fact that all sperm with a low orange/green ratio and included in Subpopulation 2 present a nucleated pattern, and no sperm included in this subpopulation showed uniform staining. Furthermore, sperm with a greater orange/green ratio and included in Subpopulation 3 does not present a nucleated pattern, but rather a more spread-out orange staining although the orange-red staining of these sperm was not uniform, but rather more intense in some localized areas of midpiece, especially at the poles. Sperm with the greatest red/orange ratio showed a more or less uniform red/orange staining in all of the midpiece. If we assume that the increase in the mitochondrial activity of a sperm cell is initiated in a low red/green JC-1 staining ratio and this ratio is progressively increased concomitantly with the increase in mitochondrial activity, we must also assume that the mitochondrial activation starts in sperm with a pattern similar to that shown in Subpopulation 2. This activation progresses to sperm with greater red/orange ratios, which implies patterns similar to that observed first as that of Subpopulation 3 and finally to that of Subpopulation 4. On the other hand, the suggestion of the existence of nucleation activating points in the midpiece would be related to the presence of mitochondria which showed a greater sensitivity to the different activation stimulus. This hypothesis would be in accordance with the results of Gaczarzewicz et al. (2003), who showed that in fresh sperm there was only a part of mitochondria within the midpiece that was fully active, while remaining mitochondria showed a reduced ability to oxidize NADH. Similarly, the activation of the apoptotic transduction signals that were concomitant with a dissipation of MMP in sperm is not carried out uniformly in all mitochondria, but there is a clear heterogeneity in the whole midpiece, thus indicating the existence of initial activation mitochondrial points from which the activation of the apoptotic transduction pathways is initiated (Krysko et al. 2001). Hence, sensitivity to external stimuli would be different in separate mitochondria from the same midpiece, reinforcing the existence of points in the midpiece with a faster and more intense response to activating factors. Of course, we are unable to follow the progressive activation of a single, specific sperm and, in this way, we are not able to confirm this hypothesis. However, the observation of the mean appearance of the sperm included in each subpopulation allows for the assumption of the nucleated mitochondrial activation hypothesis.

In conclusion, the analysis of the results obtained by both a mean-data-analysis approach and a subpopulational analysis showed the existence of significant changes in the mitochondrial activity of boar sperm subjected to IVC and subsequent IVAR. These changes were manifested by a mean increase in the oxidative metabolism after IVAR, without a concomitant mean increase in MMP. However, both IVC and IVAR activate MMP in specific sperm subpopulations with separate sensitivities, thus confirming the existence of highly heterogeneous sperm in a whole ejaculate. All of these results suggest the existence of a well-coordinated pattern in boar-sperm mitochondrial activity modulation keeping in mind the possibility of the existence of separate, specialized sperm subpopulations in 
accordance with their ability to modulate mitochondrial activation.

\section{Acknowledgements}

We thank Mr. Chuck Simmons for his accurate revision of the English grammar of this manuscript. This work has been supported by Grants AGL2004-04756-C02/02/GAN and AGL2008-01792GAN (Dirección General de Programas y Transferencia de Conocimiento, Ministerio de Ciencia e Innovación, Spain)

\section{Conflict of interest}

None of the authors have any conflict of interest to declare.

\section{Author contributions}

L Ramió-Lluch: The main investigation worker, she performed or collaborated in all of the experiments performed here. JM FernándezNovell: Responsible for all of the laser confocal microscope analysis performed here. A Peña: Responsible for the schedule and the maintenance of all of the structure needed here. C Colás, JA Cebrián-Pérez and T Muiño-Blanco: Responsible for all the experiments concerning $\mathrm{O} 2$ consumption. They also collaborate in the writing of the manuscript and the elaboration of the experimental design. A Ramírez, II Concha and T Rigau: Responsible for all of the JC-1 experiments. They also collaborate in the writing of the manuscript and the elaboration of the experimental design. JE Rodríguez-Gil: Main responsible for the experimental design, writing of the manuscript and coordination of all of the works performed here. He also obtained the funds needed for the realization of this work.

\section{References}

Aalbers JG, Mann T, Polge C, 1961: Metabolism of boar semen in relation to sperm motility and survival. J Reprod Fertil 2, 42-53.

Austin CR, 1951: Observations on the penetration of the sperm into the mammalian egg. Aust J Biol Sci 4, 581-596.

Balis UJ, Behnia K, Dwarakanath B, Bhatia SN, 1999: Oxygen consumption characteristics of porcine hepatocytes. Metab Eng 1, 49-62.

Breitbart H, Naor Z, 1999: Protein kinases in mammalian sperm capacitation and the acrosome reaction. Rev Reprod 4, 151159.

Burnette WN, 1981: 'Western blotting': electrophoretic transfer of proteins from sodium dodecyl sulfate-polyacrylamide gels to unmodified nitrocellulose and radiographic detection with antibody and radioiodinated protein A. Anal Biochem 112, 195-203.

Bussalleu E, Pinart E, Yeste M, Briz M, Sancho S, Garcia-Gil N, Badia E, Bassols J, Pruneda A, Casas I, Bonet S, 2005: Development of a protocol for multiple staining with fluorochromes to assess the functional status of boar spermatozoa. Microsc Res Tech 68, 277-283.

Chang MC, 1951: Fertilizing capacity of spermatozoa deposited into fallopian tubes. Nature 168, 697-698.

Dubé C, Leclerc P, Baba T, Reyes-Moreno C, Bailey J, 2005: The proacrosin binding protein, sp32, is tyrosine phosphorylated during capacitation of pig sperm. J Androl 26, 519-528.

Flores E, Fernández-Novell JM, Peña A, Rodríguez-Gil JE, 2009: The degree of resistance to freezing-thawing is related to specific changes in the motile sperm subpopulations structure and mitochondrial activity in boar spermatozoa. Theriogenology 72, 784-797.

Gaczarzewicz D, Piasecka M, Udala J, Blaszczyk B, Laszczynska M, Kram A, 2003: Oxidoreductive capability of boar sperm mitochondria in fresh semen and during their preservation in BTS extender. Reprod Biol 3, 161-172.

Garner DL, Thomas CA, 1999: Organellespecific probe JC-1 identifies membrane potential differences in the mitochondrial function of bovine sperm. Mol Reprod Dev 53, 222-229.

Gravance CG, Garner DL, Baumber J, Ball BA, 2000: Assessment of equine sperm mitochondrial function using JC-1. Theriogenology 53, 1691-1703.

Grunewald S, Kriegel C, Baumann T, Glander HJ, Paasch U, 2009: Interactions between apoptotic signal transduction and capacitation in human spermatozoa. Hum Reprod 24, 2071-2078.

Guthrie HD, Welch GR, Long JA, 2008 Mitochondrial function and reactive oxygen species action in relation to boar motility. Theriogenology 70, 1209-1215.

Harrison RAP, Ashworth PJC, Miller NGA, 1996: Bicarbonate $/ \mathrm{CO}_{2}$, an effector of capacitation, induces a rapid and reversible change in the lipid architecture of boar sperm plasma membranes. Mol Reprod Dev 45, 378-391.

Huo LJ, Ma XH, Yang ZM, 2002: Assessment of sperm viability, mitochondrial activity, capacitation and acrosome intactness in extended boar semen during long-term storage. Theriogenology $\mathbf{5 8}$, $1349-1360$

Jiménez I, Gónzalez-Márquez $\mathrm{H}$, Ortiz $\mathrm{R}$, Herrera JA, García A, Betancourt M, Fierro R, 2003: Changes in the distribution of lectin receptors during capacitation and acrosome reaction in boar spermatozoa. Theriogenology 59, 11711180

Jones AR, Gillan J, 1996: Glycerol 3-phosphate dehydrogenase of boar spermatozoa: inhibition by $\{$ alpha bromohydrinphosphate. J Reprod Fertil 108, 95-100

Kamp G, Büsselmann G, Jones N, Wiesner B, Lauterwein J, 2003: Energy metabolism and intracellular $\mathrm{pH}$ in boar spermatozoa. Reproduction 126, 517-525.

Kasai T, Ogawa K, Mizuno K, Nagai S, Uchida Y, Ohta S, Fujie M, Suzuki K, Hirata S, Hoshi K, 2002: Relationship between sperm mitochondrial membrane potential, sperm motility, and fertility potential. Asian J Androl 4, 97-103.

Krysko DV, Roels F, Leybaert L, D'Herde K, 2001: Mitochondrial transmembrane potential changes support the concept of mitochondrial heterogeneity during apoptosis. J Histochem Cytochem 49, 1277 1284

Laemmli UK, 1970: Cleavage of structural proteins during the assembly of the head of bacteriophage T4. Nature 227, 680-685

Mann SL, Patton WC, King A, Chan PJ 2002: Comparative genomic hybridization analysis of sperm DNA apoptosis after exposure to heat shock. J Assist Reprod Genet 19, 195-200.
Marin S, Chiang K, Bassilian S, Lee WN, Boros LG, Fernandez-Novell JM, Centelles JJ, Medrano A, Rodriguez-Gil JE, Cascante M, 2003: Metabolic strategy of boar spermatozoa revealed by metabolomic characterization. FEBS Lett 554, 342-346.

Martí E, Pérez-Pé R, Colás C, MuiñoBlanco T, Cebrián-Pérez JA, 2008: Study of apoptosis-related markers in ram spermatozoa. Anim Reprod Sci 106, 113-132.

Martínez-Pastor F, Johannisson A, Gil J, Kaabi M, Anel L, Paz P, RodríguezMartínez H, 2004: Use of chromatin stability assay, mitochondrial stain JC-1, and fluorometric assessment of plasma membrane to evaluate frozen-thawed ram semen. Anim Reprod Sci 84, 121133.

McBride HM, Neuspiel M, Wasiak S, 2006: Mitochondria: more than just a powerhouse. Curr Biol 16, R551-R560.

McLean DJ, Korn N, Pérez BS, Thurston RJ, 1993: Isolation and characterization of mitochondria from turkey spermatozoa. J Androl 14, 433-438.

Miki K, Qu W, Goulding EH, Willis WD, Bunch DO, Strader LF, Perreault SD, Eddy EM, O'Brien DA, 2004: Glyceraldehyde 3-phosphate dehydrogenase-S, a sperm-specific glycolytic enzyme, is required for sperm motility and male fertility. Proc Natl Acad Sci USA 101, 16501-16506

Mukai C, Okuno M, 2004: Glycolysis plays a major role for adenosine triphosphate supplementation in mouse sperm flagellar movement. Biol Reprod 71, 540-547.

Ramió L, Rivera MM, Ramírez A, Concha II, Peña A, Rigau T, Rodríguez-Gil JE, 2008: Dynamics of motile-sperm subpopulation structure in boar ejaculates subjected to 'in vitro' capacitation and further 'in vitro' acrosome reaction. Theriogenology $69,501-512$.

Ramió-Lluch L, Balasch S, Bonet S, Brito M, Pinart E, Rodríguez-Gil JE, 2009: Effects of filtration through Sephadex columns improve overall quality parameters and 'in vivo' fertility of subfertile refrigerated boar semen. Anim Reprod Sci 115, 189-200.

Rich PR, 2003: The molecular machinery of Keilin's respiratory chain. Biochem Soc Trans 31, 1095-1105.

Rodríguez-Martínez H, 2007: Role of the oviduct in sperm capacitation. Theriogenology 68, 138-146. 
SAS Statistical Analysis System, 1982: SAS User's Guide: Statistics. SAS Institute, Cary, NC.

Satake N, Elliot RMA, Watsom PF, Holt WV, 2006: Sperm selection and competition in pigs may be mediated by the differential motility activation and suppression of sperm subpopulations within the oviduct. J Exp Biol 209, 1560-1572.

Tardif S, Dubé C, Chevalier S, Bailey J, 2001: Capacitation is associated with tyrosine phosphorylation and tyrosine kinase-like activity of pig sperm proteins. Biol Reprod 65, 784-792.

Travert C, Carreau S, Galeraud-Denis I, 2009: In vitro capacitation. Gynecol Obstet Fertil 37, 523-528.
Urner F, Sakkas D, 2003: Protein phosphorylation in mammal spermatozoa Reproduction 125, 17-26.

Visconti PE, Kopf G, 1998: Regulation of protein phosphorylation during sperm capacitation. Biol Reprod 59, 1-6.

Wang X, Sharma RK, Gupta A, George V, Thomas AJ, Falcone T, Agarwal A, 2003 Alterations in mitochondria membrane potential and oxidative stress in infertile men: a prospective observational study. Fertil Steril 80, 844-850.

Wu JT, Chiang KC, Cheng FP, 2006: Expression of progesterone receptor(s) during capacitation and incidence of acrosome reaction induced by progester- one and zona proteins in boar spermatozoa. Anim Reprod Sci 93, 34-45.

Yanagimachi R, 1994: Mammalian fertilization. In: Knobil E, Neill JD (eds), The Physiology of Reproduction. Raven

Press, New York, Vol. 1, pp. 189-317.

Submitted: 17 May 2010; Accepted: 22 Oct 2010

Author's address (for correspondence): JE Rodríguez-Gil, Department Medicina i Cirurgia Animals, Facultat de Veterinària, Universitat Autònoma de Barcelona, E-08193 Bellaterra, Spain. E-mail: juanenrique. rodriguez@uab.cat 